\title{
Spin stiffness in the frustrated Heisenberg antiferromagnet
}

\author{
M. S. L. du Croo de Jongh and P. J. H. Denteneer \\ Instituut-Lorentz, University of Leiden, P.O. Box 9506, 2300 RA Leiden, The Netherlands
}

(Received 26 June 1996; revised manuscript received 16 October 1996)

\begin{abstract}
We calculate the spin stiffness of the $S=\frac{1}{2}$ frustrated Heisenberg antiferromagnet directly from a general formula which is evaluated in the Schwinger-boson mean-field approximation. Both Néel and collinear ordering are considered. For collinear ordering, we take the anisotropy of this phase into account, unlike previous approaches. For Néel ordering, a detailed study is made of the finite-size scaling behavior of the two terms that make up the spin stiffness. The exponents of the scaling with the system size of the two terms comprising the spin stiffness turn out to be identical to those of the unfrustrated case. [S0163-1829(97)09905-0]
\end{abstract}

\section{INTRODUCTION}

The recent interest in the frustrated Heisenberg antiferromagnets is motivated by high- $T_{c}$ superconductivity; the undoped compounds show long-range antiferromagnetic order, similar to the Heisenberg model. Upon doping, superconductivity occurs. Adding frustration to the Heisenberg model can be thought of as to mimic the effect of hole doping.

We consider the frustrated Heisenberg model on a square lattice with $N=L^{2}$ sites. It is described by the following Hamiltonian for quantum spins $\mathbf{S}_{j}$ on a lattice:

$$
H=J_{1} \sum_{\mathrm{NN}} \mathbf{S}_{i} \cdot \mathbf{S}_{j}+J_{2} \sum_{\mathrm{NNN}} \mathbf{S}_{i} \cdot \mathbf{S}_{j}
$$

where NN denotes a pair $(i j)$ of nearest-neighbor sites and NNN a pair of next-nearest-neighbor sites. The spin length is fixed, $S=\frac{1}{2}$. Both $J_{1}$ and $J_{2}$ are taken to be nonnegative. If $J_{2} / J_{1}$ is small, the antiferromagnetic long-range order is recovered (Néel-like). For $J_{2} / J_{1}$ large, the system decomposes in two Néel-ordered sublattices which, however, have the same quantization axis. Alternating strips of up and down spins will occur, the so-called collinear ordering. Clearly these couplings frustrate each other. If the spins were classical objects, a large number of phases, among which the Néel and collinear phases, would become degenerate for $J_{2} / J_{1}=0.5$. For the quantum case, a quantum phase transition to a spin-liquid phase might occur. ${ }^{1-9}$

In this paper, our intention is to employ the spin stiffness $\rho_{s}$ to measure magnetic order in the system. We calculate the spin stiffness in the framework of the Schwinger-boson mean-field approximation (SBMFA) using a general formula for $\rho_{s}$. Previous evaluations of the spin stiffness were indirect. $^{3,11,12}$ However, apart from minor adjustments, we confirm their results. Furthermore, the scaling behavior of $\rho_{s}$ in this approximation is derived. This is useful to sensibly extrapolate results of more exact approaches, like quantum Monte Carlo and exact diagonalization. Also, the typical system sizes for which scaling is valid can be estimated in this way. $^{5-7}$

\section{SCHWINGER-BOSON MEAN-FIELD APPROXIMATION}

The SBMFA improves upon standard mean-field theory by incorporating correlations between neighboring spins. ${ }^{10,11}$
With this approximation we derive the energies and wave functions of all states of the frustrated Heisenberg model for both Néel and collinear order. Our notation below will generally follow that of Mila et al. ${ }^{2}$

The Schwinger-boson transformation is a representation of the separate spin operators by pairs of boson operators, $S^{+}=a^{\dagger} b, S^{-}=a b^{\dagger}, S^{z}=\frac{1}{2}\left[a^{\dagger} a-b^{\dagger} b\right]$, supplemented by the local constraint $a^{\dagger} a+b^{\dagger} b=2 S(=1)$.

To transform the Hamiltonian in a convenient form an appropriate rotation in spin space is applied. Define $D_{i j}=a_{i} a_{j}^{\dagger}+b_{i} b_{j}^{\dagger}$ and $B_{i j}=a_{i} b_{j}+b_{i} a_{j}$. The Hamiltonian becomes

$$
H=-\frac{1}{2} \sum_{\mathrm{AFM}} J_{i j}\left(B_{i j}^{\dagger} B_{i j}-\frac{1}{2}\right)+\frac{1}{2} \sum_{\mathrm{FM}} J_{i j}\left(D_{i j}^{\dagger} D_{i j}-\frac{3}{2}\right),
$$

where we have inserted $D_{i j}$ for the pairs $(i j)$ of spins parallel in the $S^{z}$ direction and $B_{i j}$ for the antiparallel pairs. For the two orderings considered this is depicted in Fig. 1. The parameter $J_{i j}$ equals $J_{1}$ for nearest neighbors and $J_{2}$ for nextnearest neighbors. The mean-field decoupling is made using the fields $\kappa_{i j}=\frac{1}{2}\left\langle D_{i j}\right\rangle$ and $\gamma_{i j}=\frac{1}{2}\left\langle B_{i j}\right\rangle$. The local constraint $a^{\dagger} a+b^{\dagger} b=1$ is replaced by a global one and enforced by means of a Lagrange multiplier $\lambda$. After a Fourier transform from $\left(a_{i}, b_{i}\right)$ to $\left(a_{\mathbf{p}}, b_{\mathbf{p}}\right)$ and a Bogoliubov transformation from $\left(a_{\mathbf{p}}, b_{\mathbf{p}}\right)$ to $\left(\alpha_{\mathbf{p}}, \beta_{\mathbf{p}}\right)$,

$$
\begin{gathered}
a_{\mathbf{p}}=\alpha_{\mathbf{p}} \cosh \theta_{\mathbf{p}}+\beta_{\mathbf{p}}^{\dagger} \sinh \theta_{\mathbf{p}}, \\
b_{-\mathbf{p}}^{\dagger}=\alpha_{\mathbf{p}} \sinh \theta_{\mathbf{p}}+\beta_{\mathbf{p}}^{\dagger} \cosh \theta_{\mathbf{p}}, \\
\tanh 2 \theta_{\mathbf{p}}=\frac{\Delta_{\mathbf{p}}}{h_{\mathbf{p}}+\lambda},
\end{gathered}
$$

the Hamiltonian becomes

$$
H_{M F}=E_{c}+\sum_{\mathbf{p}} \omega_{\mathbf{p}}\left(\alpha_{\mathbf{p}}^{\dagger} \alpha_{\mathbf{p}}+\beta_{\mathbf{p}} \beta_{\mathbf{p}}^{\dagger}\right)
$$

where we have introduced the quasiparticle energies

$$
\omega_{\mathbf{p}}=\sqrt{\left(h_{\mathbf{p}}+\lambda\right)^{2}-\Delta_{\mathbf{p}}^{2}}
$$




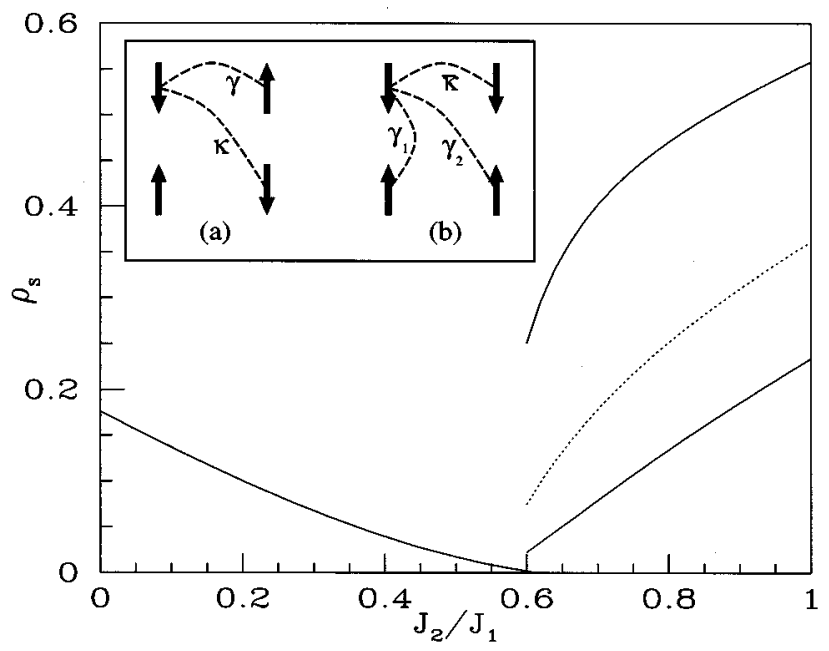

FIG. 1. The spin stiffness, $\rho_{s}$ in units of $J_{1}$ (solid lines). For the collinear ordering the spin stiffnesses in the direction of the parallel spins, $\phi=0$ (lower solid curve), and in the direction of the antiparallel spins $\phi=\pi / 2$ (upper solid curve), are drawn. The dotted line is the result found by Ivanov and Ivanov (Ref. 3). Inset : The mean fields for the Néel (a) and the collinear order (b). $\kappa \sim D_{i j}$ and $\gamma \sim B_{i j}$.

Below the quantities $h_{\mathbf{p}}, \Delta_{\mathbf{p}}$, and $E_{c}$ will be defined for the Néel and collinear orderings separately. The fields $\kappa_{i j}$ and $\gamma_{i j}$ and the Lagrange multiplier $\lambda$ are obtained through consistency equations.

We consider two types of order.

Néel order. The specific forms of $h_{\mathbf{p}}, \Delta_{\mathbf{p}}, E_{c}$ and the three consistency equations are

$$
\begin{gathered}
h_{\mathbf{p}}=4 J_{2} \kappa \cos p_{x} \cos p_{y}, \\
\Delta_{\mathbf{p}}=2 J_{1} \gamma\left(\cos p_{x}+\cos p_{y}\right), \\
E_{c}=2 N\left[J_{1}\left(\frac{1}{4}+2 \gamma^{2}\right)-J_{2}\left(\frac{3}{4}+2 \kappa^{2}\right)-\lambda\right], \\
\kappa=\frac{1}{N} \sum_{\mathbf{p}} \frac{h_{\mathbf{p}}+\lambda}{2 \omega_{\mathbf{p}}} \cos p_{x} \cos p_{y}, \\
\gamma=\frac{1}{N} \sum_{\mathbf{p}} \frac{\Delta_{\mathbf{p}}}{4 \omega_{\mathbf{p}}}\left(\cos p_{x}+\cos p_{y}\right), \\
1=\frac{1}{N} \sum_{\mathbf{p}} \frac{h_{\mathbf{p}}+\lambda}{2 \omega_{\mathbf{p}}} .
\end{gathered}
$$

For future applications it is also useful to define the "condensate'" $m_{s}$ (Ref. 13) by

$$
m_{s}=\frac{h_{\mathbf{0}}+\lambda}{N \omega_{\mathbf{0}}}=1-\frac{1}{N_{\mathbf{p} \neq(0,0),(\pi, \pi)}} \sum_{2 \omega_{\mathbf{p}}} .
$$

This is the combination of the equivalent $\mathbf{p}=(0,0)$ and $\mathbf{p}=(\pi, \pi)$ terms in Eqs. (9) and (11), which both diverge for $N \rightarrow \infty$. In the same limit, $N \rightarrow \infty, m_{s}$ also equals the corresponding terms in Eq. (10).

Collinear order. For this phase we introduce quantities with a bar where confusion might arise:

$$
\begin{gathered}
\bar{h}_{\mathbf{p}}=2 J_{1} \bar{\kappa} \cos p_{x}, \\
\bar{\Delta}_{\mathbf{p}}=2 J_{1} \gamma_{1} \cos p_{y}+4 J_{2} \gamma_{2} \cos p_{x} \cos p_{y}, \\
\bar{E}_{c}=2 N\left[J_{1}\left(\gamma_{1}^{2}-\bar{\kappa}^{2}-\frac{1}{4}\right)+J_{2}\left(\frac{1}{4}+2 \gamma_{2}^{2}\right)-\bar{\lambda}\right], \\
\bar{\kappa}=\frac{1}{N} \sum_{\mathbf{p}} \frac{\bar{h}_{\mathbf{p}}+\bar{\lambda}}{2 \bar{\omega}_{\mathbf{p}}} \cos p_{x}, \\
\gamma_{1}=\frac{1}{N} \sum_{\mathbf{p}} \frac{\bar{\Delta}_{\mathbf{p}}}{2 \bar{\omega}_{\mathbf{p}}} \cos p_{y}, \\
\gamma_{2}=\frac{1}{N} \sum_{\mathbf{p}} \frac{\bar{\Delta}_{\mathbf{p}}}{2 \bar{\omega}_{\mathbf{p}}} \cos p_{x} \cos p_{y}, \\
1=\frac{1}{N} \sum_{\mathbf{p}} \frac{\bar{h}_{\mathbf{p}}+\bar{\lambda}}{2 \bar{\omega}_{\mathbf{p}}} .
\end{gathered}
$$

The condensate $\bar{m}_{s}$ and $\bar{\omega}_{\mathrm{p}}$ are defined in a similar manner as before in Eqs. (12) and (5).

The discussion above has given us the ground states $|0\rangle$ with energy $E_{0}=E_{c}+\Sigma_{\mathbf{p}} \omega_{\mathbf{p}}$ for both orderings. These ground states are characterized by the absence of quasiparticles; $\alpha|0\rangle=\beta|0\rangle=0$. Excited states are given by

$$
\begin{gathered}
|a\rangle=\alpha_{1}^{\dagger} \times \cdots \times \alpha_{n}^{\dagger} \times \beta_{1}^{\dagger} \times \cdots \times \beta_{m}^{\dagger}|0\rangle, \\
H_{M F}|a\rangle=E_{a}|a\rangle=\left[E_{0}+\omega_{1}+\cdots+\omega_{n}+\omega_{1}+\cdots+\omega_{m}\right]|a\rangle .
\end{gathered}
$$

\section{SPIN STIFFNESS IN THE SBMFA}

The spin stiffness $\rho_{s}$ is nonzero if there exists magnetic order in the system and is, at $T=0$, associated with an increase in energy upon twisting the order parameter of the system $\left(\Delta E=\frac{1}{2} N \rho_{s}|\mathbf{q}|^{2}\right.$ with $\mathbf{q}$ the wave vector of the twist. ${ }^{15,14}$ In line with Einarsson and Schulz ${ }^{5}$ we introduce this twist through a replacement of $D_{i j}$ and $B_{i j}$ in Eq. (2) by

$$
\begin{aligned}
& D_{i j}(\mathbf{q})=a_{i} a_{j}^{\dagger} e^{i \mathbf{q} \cdot \mathbf{r}_{\delta} / 2}+b_{i} b_{j}^{\dagger} e^{-i \mathbf{q} \cdot \mathbf{r}_{\delta} / 2}, \\
& B_{i j}(\mathbf{q})=a_{i} b_{j} e^{i \mathbf{q} \cdot \mathbf{r}_{\delta} / 2}+b_{i} a_{j} e^{-i \mathbf{q} \cdot \mathbf{r}_{\delta} / 2},
\end{aligned}
$$

with $\mathbf{r}_{\delta}=\mathbf{r}_{j}-\mathbf{r}_{i}$. The resulting Hamiltonian $H(\mathbf{q})$ is now evaluated within the SBMFA.

Defining $\kappa_{i j}(\mathbf{q})=\frac{1}{2}\left\langle D_{i j}(\mathbf{q})\right\rangle$ and $\gamma_{i j}(\mathbf{q})=\frac{1}{2}\left\langle B_{i j}(\mathbf{q})\right\rangle$, the mean-field Hamiltonian becomes

$$
\begin{aligned}
H_{M F}(\mathbf{q})= & -\sum_{\mathrm{AFM}} J_{i j} \gamma_{i j}(\mathbf{q})\left(B_{i j}^{\dagger}(\mathbf{q})+B_{i j}(\mathbf{q})-2 \gamma_{i j}(\mathbf{q})\right] \\
& +\sum_{\mathrm{FM}} J_{i j} \kappa_{i j}(\mathbf{q})\left(D_{i j}^{\dagger}(\mathbf{q})+D_{i j}(\mathbf{q})-2 \kappa_{i j}(\mathbf{q})\right] \\
& +\lambda \sum_{i}\left(a_{i}^{\dagger} a_{i}+b_{i}^{\dagger} b_{i}-1\right)+\text { constants. }
\end{aligned}
$$

Since within the SBMFA we know what the excited states are [see Eqs. (19) and (20)], the spin stiffness can be directly evaluated from second-order perturbation theory: 


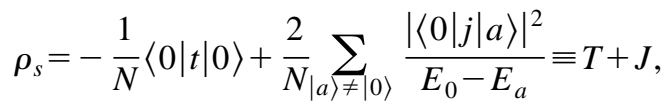

with the quantities $t$ and $j$ defined by

$$
t=-\left.\frac{d^{2}}{d q^{2}} H_{M F}(\mathbf{q})\right|_{\mathbf{q}=0}, \quad j=\left.\frac{d}{d q} H_{M F}(\mathbf{q})\right|_{\mathbf{q}=0} .
$$

In Eq. (24), we have also defined the abbreviations $T$ and $J$ for the two terms in $\rho_{s}$.

\section{RESULTS FOR THE SPIN STIFFNESS}

From this point on, we set $\mathbf{q}=q(\cos \phi, \sin \phi)$. Of the two terms for $\rho_{s}$ in Eq. (24), $T$ is evaluated more easily. We obtain after straightforward manipulations with Brillouin zone summations,

$$
\begin{gathered}
T=J_{1} \gamma^{2}-2 J_{2} \kappa^{2}, \\
\bar{T}=2 J_{2} \gamma_{2}^{2}+J_{1}\left(\gamma_{1}^{2} \sin ^{2} \phi-\bar{\kappa}^{2} \cos ^{2} \phi\right) .
\end{gathered}
$$

These simple equations hold for all system sizes $N$.

The quantity $J$ requires more effort; the operator $j$ has to be expressed in the operators $\alpha_{\mathbf{p}}$ and $\beta_{\mathbf{p}}$ defined in Eq. (3). For the wave functions $|a\rangle$ and energies $E_{a}$ of the excitations we use Eqs. (19) and (20). The resulting values for $J$ are written as summations over the Brillouin zone:

$$
\begin{gathered}
J=-\frac{1}{N} \sum_{\mathbf{p}} \frac{\sin ^{2} p_{y}}{\omega_{\mathbf{p}}^{3}}\left[J_{1} \gamma\left(h_{\mathbf{p}}+\lambda\right)-2 J_{2} \kappa \Delta_{\mathbf{p}} \cos p_{x}\right]^{2}, \\
\bar{J}=-\frac{1}{N} \sum_{\mathbf{p}} \frac{1}{\bar{\omega}_{\mathbf{p}}^{3}}\left\{\cos \phi \sin p_{x}\left[J_{1} \bar{\kappa}_{\mathbf{p}}-2 J_{2} \gamma_{2}\left(\bar{h}_{\mathbf{p}}+\bar{\lambda}\right) \cos p_{y}\right]\right. \\
\left.-\sin \phi \sin p_{y}\left[J_{1} \gamma_{1}\left(\bar{h}_{\mathbf{p}}+\bar{\lambda}\right)+2 J_{2} \gamma_{2}\left(\bar{h}_{\mathbf{p}}+\bar{\lambda}\right) \cos p_{x}\right]\right\}^{2} .
\end{gathered}
$$

Only for the infinitely large lattice can these equations be simplified by replacing summations by integrals and partially integrating. The expression for the spin stiffness $\rho_{s}=T+J$ then simplifies considerably and becomes

$$
\begin{gathered}
\rho_{s}=m_{s}\left(J_{1} \gamma-2 J_{2} \kappa\right), \\
\bar{\rho}_{s}=\bar{m}_{s}\left[\left(2 J_{2} \gamma_{2}-J_{1} \bar{\kappa}\right) \cos ^{2} \phi+\left(2 J_{2} \gamma_{2}+J_{1} \gamma_{1}\right) \sin ^{2} \phi\right] .
\end{gathered}
$$

Ivanov and Ivanov ${ }^{3}$ apply a different method to derive $\rho_{s}$. They use a modified spin-wave theory which leads to the same consistency equations as the Schwinger-boson approach. $\rho_{s}$ is then obtained by calculating the correlation length $\xi$ associated with the spin-spin correlation function $\left\langle\mathbf{S}_{i} \cdot \mathbf{S}_{j}\right\rangle$ and comparing this $\xi$ to the expression for $\xi$ obtained for the nonlinear $\sigma$ model to two-loop order by Chakravarty et al. ${ }^{12}$ [where $\xi \sim \exp \left(2 \pi \rho_{s} / \theta\right)$, with $\theta$ the temperature]. For Néel ordering their expression is identical to our result (28). It is gratifying to see that the nonlinear $\sigma$ model also is the effective field theory for the low-energy physics of the frustrated Heisenberg antiferromagnet.

For the collinear ordering they obtain the geometrical average of our $\cos ^{2} \phi$ and $\sin ^{2} \phi$ terms, whereas we take explic-
TABLE I. The limit $(N=\infty)$ values for $T,-J$, and $\rho_{s}$ as function of the ratio $J_{2} / J_{1}$ where $J_{1}=1$. The two orderings considered are Néel $(\mathrm{N})$ and collinear $(\mathrm{C})$ order. For the collinear ordering there are two directions: along the antiparallel spins $\phi=\pi / 2$ (listed first) and along the parallel spins $\phi=0$ (listed second).

\begin{tabular}{lccccccc}
\hline \hline$J_{2} / J_{1}$ & Order & $T$ & & $-J$ & & $\rho_{s}$ & \\
\hline 0.0 & $\mathrm{~N}$ & 0.3352 & & 0.1596 & & 0.1757 & \\
0.1 & $\mathrm{~N}$ & 0.2961 & & 0.1596 & & 0.1365 & \\
0.2 & $\mathrm{~N}$ & 0.2597 & & 0.1597 & & 0.1000 & \\
0.3 & $\mathrm{~N}$ & 0.2271 & & 0.1600 & & 0.0672 & \\
0.4 & $\mathrm{~N}$ & 0.1995 & & 0.1604 & & 0.0391 & \\
0.5 & $\mathrm{~N}$ & 0.1783 & & 0.1612 & & 0.0171 & \\
0.6 & $\mathrm{~N}$ & 0.1639 & & 0.1622 & & 0.0017 & \\
0.6 & $\mathrm{C}$ & 0.6231 & 0.0499 & 0.3841 & 0.0284 & 0.2390 & 0.0214 \\
0.7 & $\mathrm{C}$ & 0.7211 & 0.1563 & 0.3327 & 0.0797 & 0.3884 & 0.0766 \\
0.8 & $\mathrm{C}$ & 0.7794 & 0.2566 & 0.3232 & 0.1264 & 0.4563 & 0.1302 \\
0.9 & $\mathrm{C}$ & 0.8327 & 0.3492 & 0.3292 & 0.1689 & 0.5034 & 0.1803 \\
1.0 & $\mathrm{C}$ & 0.8866 & 0.4361 & 0.3434 & 0.2086 & 0.5432 & 0.2275 \\
1.1 & $\mathrm{C}$ & 0.9420 & 0.5190 & 0.3623 & 0.2465 & 0.5797 & 0.2725 \\
\hline \hline
\end{tabular}

itly the anisotropy of this phase into account. Still both expressions for $\overline{\rho_{s}}$ vanish at the same value of $J_{2} / J_{1}$.

Table I and Fig. 1 contain our numerical results.

\section{SCALING OF THE SPIN STIFFNESS}

It is necessary to know the size dependence of observables to obtain a good approximation for their limit values. Neuberger and Ziman ${ }^{16}$ derived the scaling behavior for an unfrustrated Heisenberg antiferromagnet explicitly. Here we extend this to the case of frustration.

Recently some discussion has arisen about where the scaling behavior of $\rho_{s}$ sets in. ${ }^{6}$ Our formulas in the last section lend themselves well to investigate this.

Here we only treat the Néel ordering. We want to know the scaling behavior of the condensate $m_{s}$ and the two terms $J$ and $T$ that make up $\rho_{s}\left(\rho_{s}=T+J\right)$. The latter two will turn out to have different scaling behavior.

As can be seen from Eq. (26) only the scaling behavior of $\kappa_{N}$ and $\gamma_{N}$ is required for $T$. These two are part of the set $\left(\kappa_{N}, \gamma_{N}, \lambda_{N}\right)$ of mutually dependent quantities. We will now argue what is the exponent of their scaling behavior and therefore of $T$, without trying to obtain the precise prefactor (which would be quite tedious).

Name Eqs. (9), (10), and (11) I, II, and III, respectively. They contain poles at $\mathbf{p}=(0,0)$ and $\mathbf{p}=(\pi, \pi)$. With help of Eqs. (5), (6), and (7) we rearrange them as I-III, II-III, and $4 J_{2} \kappa_{N} \mathrm{I}-4 J_{1} \gamma_{N} \mathrm{II}+\lambda_{N} \mathrm{III}$. We neglect the $\mathbf{p}=(0,0)$ and $\mathbf{p}=(\pi, \pi)$ terms in the summations. It is easy to show that this will give rise to errors of the order $O\left(N^{-2}\right)$. Next we expand these equations to first order around their infinite-size values $(\kappa, \gamma, \lambda)$. Define the size dependences $\delta \kappa_{N}=\kappa_{N}-\kappa$, $\delta \gamma_{N}=\gamma_{N}-\gamma$, and $\delta \lambda_{N}=\lambda_{N}-\lambda$ to obtain the equation

$$
\left[\begin{array}{c}
\kappa-1 \\
\gamma-1 \\
\lambda-4 J_{1} \gamma^{2}+4 J_{2} \kappa^{2}
\end{array}\right]=\vec{A}+\overrightarrow{\vec{B}} \cdot\left[\begin{array}{c}
\delta \kappa_{N} \\
\delta \gamma_{N} \\
\delta \lambda_{N}
\end{array}\right],
$$


where $\vec{A}$ and $\vec{B}$ contain summations over the Brillouin zone dependent on the infinite-size parameters $\kappa, \gamma$, and $\lambda$. The remaining size dependence of $\overrightarrow{\vec{B}}$ can be neglected as it leads to higher-order terms. On the other hand, the summations in $\vec{A}$ will be replaced by integrations plus size-dependent corrections. Using Neuberger and Ziman ${ }^{16}$ we obtain

$$
\vec{B} \cdot\left[\begin{array}{l}
\delta \kappa_{N} \\
\delta \gamma_{N} \\
\delta \lambda_{N}
\end{array}\right]=\frac{1}{N^{3 / 2}} \vec{C} .
$$

The parameters $\kappa_{N}, \gamma_{N}$, and $\lambda_{N}$ thus scale with $N^{-3 / 2}$. A direct consequence of this is that $T_{N}-T \sim N^{-3 / 2}$. If the size dependence of the parameters is neglected $\left[\left(\kappa_{N}, \gamma_{N}, \lambda_{N}\right) \rightarrow(\kappa, \gamma, \lambda)\right.$ inside the summations (9)-(11)], we also find the prefactor

$$
T_{N}-T=\frac{0.7186}{N^{3 / 2}} \frac{1}{\sqrt{2}} \sqrt{\frac{\lambda+4 J_{2} \kappa}{\lambda-4 J_{2} \kappa}}\left[\lambda-8 J_{2} \kappa\right] .
$$

Up to $J_{2} / J_{1} \approx 0.5$ this is in good agreement with the numerical solution of Eqs. (9)-(11) for various sizes $N$.

Next we consider the condensate $m_{s, N}$ and $J_{N}$, for which we can even derive the prefactors. Replacement of $\left(\kappa_{N}, \gamma_{N}, \lambda_{N}\right)$ by $(\kappa, \gamma, \lambda)$ in the summation of Eq. (12) leads to errors of the order $O\left(N^{-3 / 2}\right)$. We will neglect these. Neuberger and Ziman ${ }^{16}$ state a lemma applicable to this summation. This leads to

$$
m_{s, N}-m_{s}=\frac{0.6208}{\sqrt{N}} \sqrt{2} \sqrt{\frac{\lambda+4 J_{2} \kappa}{\lambda-4 J_{2} \kappa}} .
$$

For $J_{N}$ we find in a similar fashion

$$
J_{N}-J=\frac{0.6208}{\sqrt{N}} \frac{\sqrt{\lambda^{2}-\left(4 J_{2} \kappa\right)^{2}}}{4 \sqrt{2}} .
$$

These formulas are in excellent agreement with the numerical solution to Eqs. (9)-(11). For $J_{N}$ this is depicted in Fig. 2.

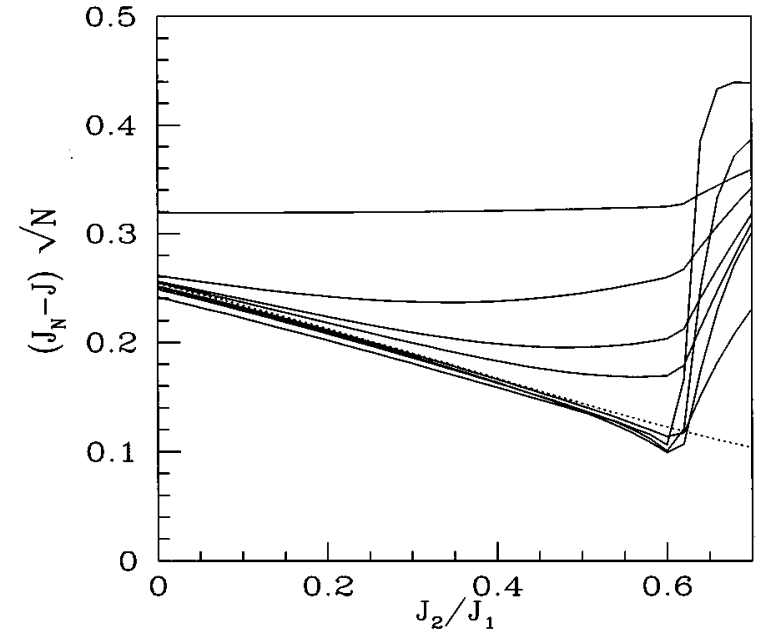

FIG. 2. The numerical scaling behavior of $J_{N}$ (solid lines) for sizes $L=2,4,6,8,10,20,40,100$ (numbering is top-down) compared with the theoretical formula (34) (dotted line).

In conclusion, we have obtained the scaling behavior of $T$ and $J$ from an analysis of the formulas in the SBMFA for the frustrated Heisenberg antiferromagnet. The qualitative scaling behavior (i.e., the exponents) is the same as for the unfrustrated case, which was discussed by Neuberger and Ziman. ${ }^{16}$ The scaling behavior that was utilized by Einarsson and Schulz ${ }^{5}$ does agree with our findings but we confirm the message of Feiguin et al. ${ }^{6}$ that the clusters they used are too small for the scaling behavior of $J$ to have set in. Numerically we see that the scaling behavior starts around size $N=100$ (see Fig. 2) whereas the largest cluster they used is $N=36$.

\section{ACKNOWLEDGMENTS}

We would like to thank J.M.J. van Leeuwen and W. van Saarloos for fruitful discussions.
${ }^{1}$ P. Chandra and B. Doucot, Phys. Rev. B 38, 9335 (1988).

${ }^{2}$ F. Mila, D. Poilblanc, and C. Bruder, Phys. Rev. B 43, 7891 (1991).

${ }^{3}$ N.B. Ivanov and P. Ch. Ivanov, Phys. Rev. B 46, 8206 (1992).

${ }^{4}$ A.V. Dotsenko and O.P. Sushkov, Phys. Rev. B 50, 13821 (1994).

${ }^{5}$ T. Einarsson and H.J. Schulz, Phys. Rev. B 51, 6151 (1995).

${ }^{6}$ A.E. Feiguin, G.J. Gazza, A.E. Trumper, and H.A. Ceccatto, Phys. Rev. B 52, 15043 (1995).

${ }^{7}$ H.J. Schulz, T.A.L. Ziman, and D. Poilblanc, J. Phys. (France) 6, 675 (1996).

${ }^{8}$ O.P. Sushkov (unpublished).

${ }^{9}$ M. Zhitomirsky and K. Ueda, Phys. Rev. B 54, 9007 (1996)
${ }^{10}$ D.P. Arovas and A. Auerbach, Phys. Rev. B 38, 316 (1988); A. Auerbach and D.P. Arovas, Phys. Rev. Lett. 61, 617 (1988).

${ }^{11}$ A. Auerbach, Interacting Electrons and Quantum Magnetism (Springer, New York, 1994).

12 S. Chakravarty, B.I. Halperin, and D.R. Nelson, Phys. Rev. B 39, 2344 (1988).

${ }^{13}$ J.E. Hirsch and S. Tang, Phys. Rev. B 39, 2850 (1989).

${ }^{14}$ M.E. Fisher, M.N. Barber, and D. Jasnow, Phys. Rev. A 188, 1111 (1973).

${ }^{15}$ J.M.J. van Leeuwen, M.S.L. du Croo de Jongh, and P.J.H. Denteneer, J. Phys. A 29, 41 (1996).

${ }^{16}$ H. Neuberger and T. Ziman, Phys. Rev. B 39, 2608 (1989). 